\title{
Study on Bitcoin Risk Perception Based on EFA and Structural Equation Model
}

\author{
Jing $\mathrm{Li}^{1, \mathrm{a},{ }^{*}}$,Qin Qin ${ }^{2, \mathrm{~b}}$, Yu Zhang ${ }^{3, \mathrm{c}}$ \\ ${ }^{1}$ College of e-business management, Wuhan International Trade University, Wuhan, Hubei,China \\ ${ }^{2}$ College of e-business management, Wuhan International Trade University, Wuhan, Hubei,China \\ ${ }^{3}$ College of economic management, Wuhan International Trade University, Wuhan, Hubei,China \\ a495579526@qq.com, b23161920@qq.com, c6270089@qq.com \\ * Jing Li
}

Keywords: Bitcoin ,Risk Perception,EFA,Structural Equation Model.

Abstract. This document established the main factors of bitcoin risk perception based on the explorative factor analysis, then studied them using structural equation model and conducted one-order and second-order confirmatory factor analysis. The results show that the bitcoin perceived risk mainly includes four aspects: bitcoin technology and security risk, national policy and legal risks, social risks, bitcoin market and transaction risk, the risk perception has correlation on each other. Based on the model, the author put forward the corresponding policy recommendations.

\section{基于EFA和结构方程模型的比特币风险感知评价分析}

\author{
李靖 ${ }^{1, a *}$, 秦琴 ${ }^{2, b}$, 张钰 ${ }^{3, c}$ \\ ${ }^{1}$ 武汉商贸职业学院电商管理学院, 武汉, 湖北, 中国 \\ 2 武汉商贸职业学院电商管理学院, 武汉, 湖北, 中国 \\ 3 武汉商贸职业学院经济管理学院, 武汉, 湖北, 中国

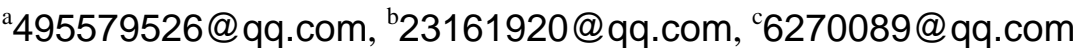 \\ *李靖
}

关键词:比特币；风险感知；探索性因子分析（EFA）；结构方程模型

中文摘要：基于探索性因子分析得到了比特币风险感知的主要因素, 并采用结构方程模型对 其进行了一阶和二阶验证性因子分析。结果表明，比特币风险感知主要有四个方面：比特币 技术与安全风险、国家政策和法律风险、社会风险以及比特币市场与交易风险, 各风险感知 之间具有相关关系。并在所建模型基础上提出了相应的政策建议。

1. 引言

近几年，随着比特币在全球范围内的风生水起，比特币市场化程度越来越高，民众的参 与度越来越广泛，加上中产阶级的崛起和投资需求的释放导致比特币成为一种新型的互联网 金融投资品甚至投机品，他们在各大比特币交易平台投放资金以期获得高额收益。然而，作 为互联网背景下诞生的一种新型事物, 比特币在其发展过程中存在着很多不确定因素, 面临 着政策风险、法律风险、信用风险、交易风险、网络安全风险、洗钱和非法交易风险等诸多

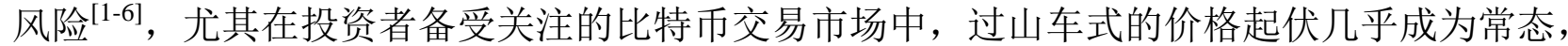


加上投资者社会、文化背景的多元化，更加导致投资者面临着许多未知的风险，投资者对于 这些风险的感知和识别存在主观性、模糊性、时变性、复杂性等特点，从而直接影响投资者 的投资策略和投资收益。

对于比特币风险感知的研究，不仅可以描绘投资者风险感知的影响因素，量化投资者风 险识别水平, 帮助投资者树立客观的比特币风险防范意识, 而且可以丰富比特币风险管理和 风险决策的理论体系，为相关部门以及比特币企业制定比特币投资风险管理策略提供参考， 帮助其逐步建立全面的风险管理体系，进而降低投资者的投资风险。

目前国内外学者的研究大都聚焦在比特币风险方面, 对于这些风险的感知研究鲜见, 尤 其是采用定性与定量相结合的方法对风险感知开展研究几乎是空白。基于以上背景, 本文将 从比特币投资者这个微观角度, 开展比特币投资者的问卷调查, 并采用探索性因子分析以及 结构方程模型的方法, 对比特币风险感知进行实证研究, 并提出应对策略, 以期对比特币投 资者和相关部门提供一定的参考。

\section{2. 比特币风险感知指标体系}

本研究主要通过两个步骤来建立比特币风险感知的指标体系。首先, 对近几年国内外比 特币风险的研究文献进行搜集、整理、归类和分析, 构建全面的比特币风险体系指标, 并把 这些风险指标作为风险感知指标的备选项; 然后, 对 12 名专家和比特币资深投资者进行访谈, 对风险感知指标的备选项进行二次笁选及补充，从国家层面、比特币自身层面、投资者个人 主观层面、交易平台和市场层面、社会层面等多个层面综合考虑, 确定了 21 个风险感知评价 指标, 分别是：政策风险（X1）、法律风险（X2）、比特币硬件技术风险（X3）、挖矿体 系崩坍风险（X4）、比特币算法技术风险（X5）、比特币相关软件技术风险（X6）、投资 者操作失误风险（X7）、投资者忘记密码风险（X8）、比特币被盗风险（X9）、比特币市 场价格风险（X10）、企业跑路或倒闭风险（X11）、企业准备金短缺风险（X12）、企业把 资金挪为他用风险（X13）、企业技术或安全风险（X14）、企业服务不及时到位引发的风险

（X15）、比特币企业篡改数据风险（X16）、比特币通货紧缩风险（X17）、比特币受山寨 币冲击风险（X18）、比特币被替代风险（X19）、洗钱或非法交易风险（X20）、自然灾害 引发的风险（X21）。

\section{3. 数据调研与探索性因子分析}

\section{1 问卷设计与数据获取}

根据以上风险感知体系指标设计比特币风险感知问卷, 选项采用李克特的 5 级评分标准, 按照风险感知从低到高分数分别记为 1-5 分。考虑到比特币投资者的分散性、特殊性和小众 性, 采用与比特币行业合作的网络调研模式, 委托国内最大的比特币交易平台 okcoin 做问卷 的上线、推广、回收工作, 精准定位调研对象。问卷回收 560 份, 有效问卷 528 份, 有效率 为 $94.3 \%$ 。

\section{2 探索性因子分析}

从整体数据样本中选取前 250 条数据, 利用 SPSS22 软件对调研结果进行探索性因子分析, 获取比特币风险感知的主要因素, 找出理论模型中的潜在变量。探索性因子分析的 KMO 值为 0.931, Bartlett' $\mathrm{s}$ 球形检验值为 6536.695 , P 值为零, 表明该数据样本适合做因子分析。 删除数据分析中因子载荷值较小的项, 最终所选因子能代表的初始信息百分比都超过 $60 \%$ 。 因子分析模型的共同度如下表所示。 
表1 因子分析模型的共同度

\begin{tabular}{|c|c|c||c|c|c||c|c|c|}
\hline 因子 & 起始 & 提取 & 因子 & 起始 & 提取 & 因子 & 起始 & 提取 \\
\hline $\mathrm{X} 1$ & 1.000 & .679 & $\mathrm{X} 9$ & 1.000 & .695 & $\mathrm{X} 15$ & 1.000 & .853 \\
\hline $\mathrm{X} 2$ & 1.000 & .684 & $\mathrm{X} 10$ & 1.000 & .798 & $\mathrm{X} 17$ & 1.000 & .799 \\
\hline $\mathrm{X} 3$ & 1.000 & .628 & $\mathrm{X} 11$ & 1.000 & .811 & $\mathrm{X} 18$ & 1.000 & .780 \\
\hline $\mathrm{X} 6$ & 1.000 & .725 & $\mathrm{X} 12$ & 1.000 & .843 & $\mathrm{X} 20$ & 1.000 & .670 \\
\hline $\mathrm{X} 7$ & 1.000 & .676 & $\mathrm{X} 14$ & 1.000 & .816 & & & \\
\hline
\end{tabular}

经过软件进行分析，输出 4 个主因子可以解释 14 个变量的累计方差为 $74.649 \%$, 即这四 个因子可以解释 $74.649 \%$ 的原始信息。用最大方差法建立旋转后的因子分析模型, 旋转后的 因子载荷矩阵如表 2 所示。

表 2 旋转后的因子载荷矩阵

\begin{tabular}{|c|c|c|c|c||c|c|c|c|c|}
\hline \multirow{2}{*}{ 指标 } & \multicolumn{4}{|c|}{ 成分 } & \multirow{2}{*}{ 指标 } & \multicolumn{4}{|c|}{ 成分 } \\
\cline { 2 - 7 } & 1 & 2 & 3 & 4 & & 1 & 2 & 3 & 4 \\
\hline $\mathrm{X} 1$ & .036 & .006 & .822 & .03 & $\mathrm{X} 11$ & .857 & .214 & .152 & .088 \\
\hline $\mathrm{X} 2$ & .241 & .152 & .775 & .039 & $\mathrm{X} 12$ & .900 & .153 & .097 & .020 \\
\hline $\mathrm{X} 3$ & .264 & .538 & .516 & -.054 & $\mathrm{X} 14$ & .868 & .192 & .128 & .071 \\
\hline $\mathrm{X} 6$ & .246 & .806 & .040 & .114 & 15 & .909 & .128 & .090 & .09 \\
\hline $\mathrm{X} 7$ & .284 & 77 & -022 & .087 & $\mathrm{X} 17$ & .10 & .160 & .011 & .872 \\
\hline $\mathrm{X} 9$ & .095 & .793 & .135 & .199 & $\mathrm{X} 18$ & .059 & .279 & .042 & .835 \\
\hline $\mathrm{X} 10$ & .570 & .563 & .276 & .281 & $\mathrm{X} 20$ & .037 & .264 & .120 & .766 \\
\hline
\end{tabular}

根据旋转后的因子载荷矩阵分析得到各个主要因子明确的含义: 因子 X1、X2 可以命名为 国家层面的风险感知因子, X3、X6、X7、X9 可以命名为技术和安全层面的风险感知因子, X10、 X11、X12、X14、X15 可以命名为市场与交易层面的风险感知因子，X17、X18、X20 可以命名 为比特币的社会风险感知因子。

\section{4. 比特币风险感知的结构方程模型}

\section{1 结构方程模型简介 ${ }^{[7]}$}

结构方程模型是一种基于变量的协方差矩阵对变量之间关系进行分析的统计方法, 它融 合了路径分析、因子分析两种统计技术。结构方程模型通常由测量模型和结构模型两个部分 组成。测量模型也被称为验证性因子分析模型, 反映潜变量及其指标变量之间的关系, 结构 模型又称作潜变量因果关系模型，反映的是潜变量之间相互影响的关系。

模型中各变量间的关系可以表示以下 3 个方程式, 其中 (1) 为结构模型方程式, (2)、(3) 为测量模型方程式。

$$
\left\{\begin{array}{c}
\eta=\mathrm{B} \eta+\Gamma \xi+\zeta \\
\mathrm{Y}=\Lambda_{\mathrm{y}} \eta+\varepsilon \\
\mathrm{X}=\Lambda_{\mathrm{x}} \xi+\delta
\end{array}\right.
$$

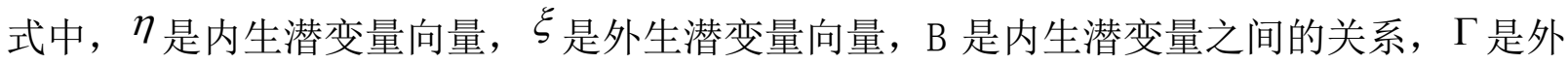
生潜变量对内生潜变量的影响系数矩阵, $\zeta$ 是随机误差项矩阵。X 是外生指标组成的向量, $\mathrm{Y}$ 是内生指标组成的向量, $\Lambda_{\mathrm{x}} 、 \Lambda_{\mathrm{y}}$ 是指标变量 $(\mathrm{X} 、 \mathrm{Y})$ 的因素负荷量, $\delta 、 \varepsilon$ 分别是 X、Y 的 随机误差组成的向量, 反映方程中未能被解释的部分。 


\section{2 一阶验证性因子分析}

\section{2.1 模型假设及构建}

以上通过对比特币风险感知进行探索性 因子分析, 得到 4 个方面的因子对应 14 个观 测变量, 所以可以假定比特币风险感知的结 构方程模型是由 4 个潜在变量构成, 它们分 别对应不同的观测变量, 所有观测变量的值 来自调研问卷中的数据。并规定 ${ }^{[8]}$ : 第一, 每 个条目在其测量的一级因子上都有一个非 0 的负荷量, 而对其他的一级因子的负荷量为 0 ；第二，误差项彼此不相关。根据确定的潜 在变量和观测变量, 构建比特币风险感知的 结构模型, 运用 Amos22 软件进行绘图和运算, 并根据 Amos 提供的修正指数, 将某些误差变 量之间的固定参数释放为自由参数, 最终得 到比特币风险感知结构方程模型的路径系数 图如图 1 所示。

\section{2.2 模型拟合结果}

模型拟合结果如表 3 所示。从表中看出, 卡方对应的 P 值具有统计显著性, 卡方与自 由度之比为 1.744 , 小于 $2, \mathrm{GFI} 、 \mathrm{AGFI}$ 、NFI、 RFI、IFI、TLI、CFI 都大于 0.9 ， RMSEA 小于 0.8 ,

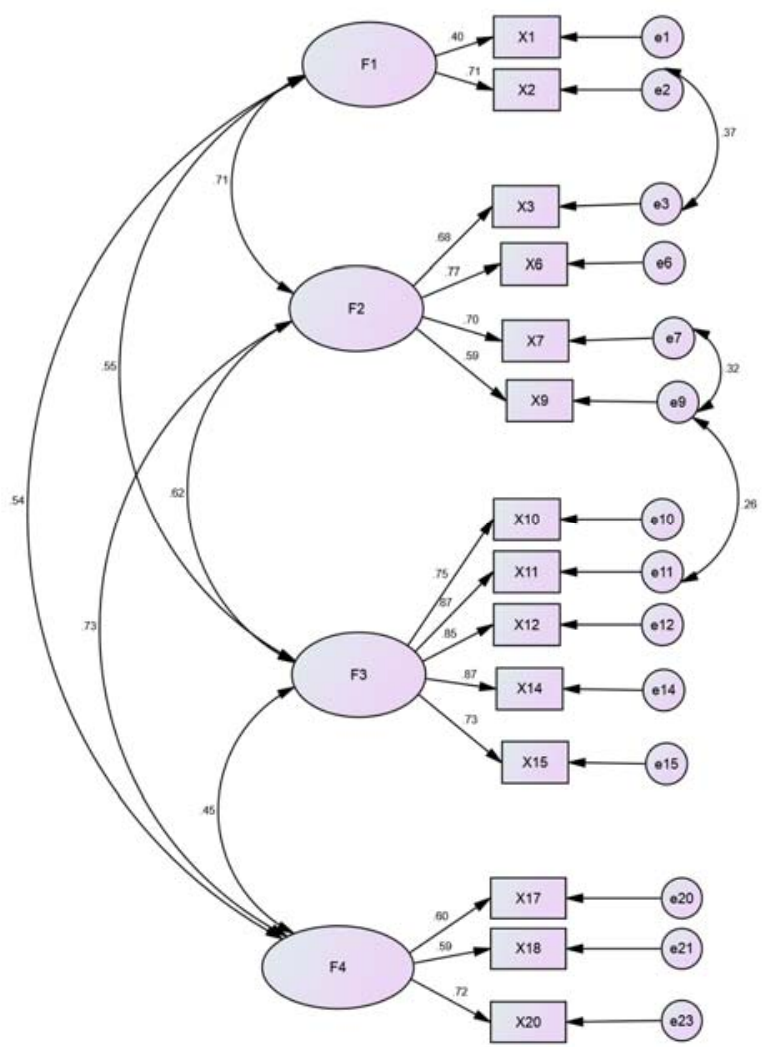

图1 一阶CFA模型路径系数图 (标准化解) 各项指标都满足模型检验与拟合优度的要求。所以，得到的比特币风险感知结构方程模型是 可以接受的。

表3 一阶CFA模型的拟合效果指标

\begin{tabular}{|c|c|c|c|c|c|c|c|c|c|c|c|}
\hline 拟合指数 & P & CMIN/DF & RMR & GFI & AGFI & NFI & RFI & IFI & TLI & CFI & RMSEA \\
\hline 拟合值 & 0.000 & 1.744 & 0.050 & 0.942 & 0.910 & 0.935 & 0.912 & 0.971 & 0.961 & 0.971 & 0.052 \\
\hline
\end{tabular}

\section{2.3 模型结果分析}

（1）潜在变量间相互关系的分析

潜在变量间相互关系的分析结果如表 4 所示。从表中数据可以看出，比特币技术安全风 险感知与比特市场交易风险感知的路径系数最高, 为 $0.726, \mathrm{t}$ 值为 5.897 , 说明比特技术安 全风险感知与比特市场交易风险感知之间具有显著的正向关系; 其次是国家层面的风险感知 与比特币技术安全风险感知的路径系数为 0.712 , $\mathrm{t}$ 值为 4.128 , 说明国家层面的风险感知与 比特币技术安全风险感知之间也具有显著的正向关系; 比特币技术安全风险感知与比特币市 场交易风险感知的路径系数为 0.616 , 国家层面风险感知与比特币市场交易风险感知、社会 风险感知的路径系数分别为 $0.547 、 0.543$, 系数值均在 0.5 以上, 均具有较显著的正向关系; 而比特市场交易风险感知与比特币社会风险感知的路径系数最低, 系数值为 $0.446, \mathrm{t}$ 值为 6. 456, 说明比特币技术安全层面的风险感知与比特币市场交易风险感知具有正向关系, 但是 相关性不高。 
表4 潜在变量间的路径系数

\begin{tabular}{|c|c|c|c|}
\hline 假设 & Estimate & $\mathrm{T}$ 值 & $\mathrm{P}$ \\
\hline $\mathrm{F} 1<-->\mathrm{F} 2$ & 0.712 & 4.128 & $* * *$ \\
\hline $\mathrm{F} 2\langle-->\mathrm{F} 3$ & 0.616 & 6.456 & $* * *$ \\
\hline $\mathrm{F} 3\langle-->\mathrm{F} 4$ & 0.446 & 4.803 & $* * *$ \\
\hline $\mathrm{F} 2<-->\mathrm{F} 4$ & 0.726 & 5.897 & $* * *$ \\
\hline $\mathrm{F} 1<-->\mathrm{F} 4$ & 0.543 & 3.586 & $* * *$ \\
\hline $\mathrm{F} 1<-->\mathrm{F} 3$ & 0.547 & 3.946 & $* * *$ \\
\hline
\end{tabular}

（2）潜在变量与观测变量间的关系分析

1)国家层面风险感知与观测变量之间的关系。国家层面风险感知有 2 个观测指标，其中 法律风险感知的系数为 0.71 , 而国家政策风险感知的系数仅为 0.40 , 说明比特币投资者对于 法律风险感知度和警惕度高于国家的政策风险。

(2)比特币技术与安全风险感知与观测量之间的关系。比特币技术与安全风险感知有 4 个 观测变量, 比特币硬件技风险感知系数最高 (0.88), 其次是比特币相关软件技术风险 $(0.77)$, 说明比特币的技术是投资者们关注的重要问题，也是风险感知的重要方面; 投资者操作失误 风险感知系数为 0.70 , 说明投资者对于比特币这项新技术的掌握和应用有较高的风险意识; 比特币被盗风险感知系数为 0.59 ，最低，说明投资者对被盗风险感知较低。

(3)比特币市场交易风险感知与观测量之间的关系。比特币市场与交易风险感知的观测量 中, 企业的跑路、倒闭风险和企业技术、安全风险的系数最高, 均为 0.87 , 其次是企业准备 金短缺风险为 0.85 , 企业服务不到位引发的风险系数为 0.73 , 以上四个观测指标都与比特币 的交易平台有紧密联系，说明投资者对于企业平 台的各种风险感知度较高; 比特币的价格风险系 数则远低于比特币交易平台带来的各种交易风险 的平均值，说明投资者对交易平台的风险感知高 于对市场的风险感知。

(4)比特币社会风险与观测量之间的关系。在 比特币社会风险感知的三个观测指标中，洗钱及 非法交易风险感知系数最高 $(0.72)$ ，比特币通货 紧缩风险为 0.60 , 比特币受山寨币冲击风险为 0.59 ，说明洗钱和非法交易是投资者关注的最重 要的社会风险。

\section{3 二阶验证性因子分析}

\section{3.1 模型假设及构建}

根据一阶 CFA 分析结果显示, 4 个潜变量 的相关系数在 $0.45-0.73$ 之间, 该模型可能具有 二阶因子结构。模型假设为：（1）比特币风险感 知可以由一个高阶共同因子和四个一阶因子解 释;（2）每个题目都对应单一因子，没有横跨的 现象; (3) 误差项之间独立无关。运用 AMOS22 绘 制二阶 CFA 模型的路径图, 如图 2 所示。

\section{3.2 模型拟合结果}

二阶 CFA 模型拟合结果见表 5。从表 5 可知，除 RMR

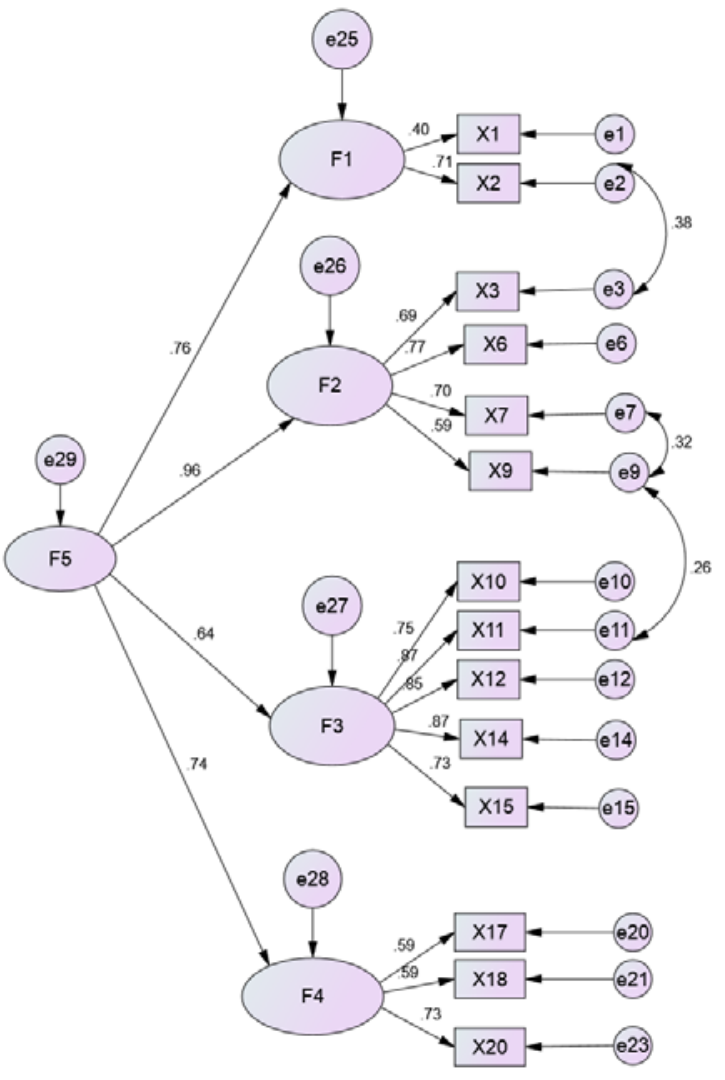

图2 二阶CFA模型路径系数图 (标准化解) 的值为 0.052 , 略大于 0.05 以外, 其他指标的值均表明模型适配度良好。 
表5 二阶CFA模型的拟合效果指标

\begin{tabular}{|c|c|c|c|c|c|c|c|c|c|c|c|}
\hline $\begin{array}{c}\text { 拟合指 } \\
\text { 数 }\end{array}$ & $\mathrm{P}$ & CMIN/DF & RMR & GFI & AGFI & NFI & RFI & IFI & TLI & CFI & RMSEA \\
\hline 拟合值 & 0.000 & 1.724 & 0.052 & 0.940 & 0.910 & 0.933 & 0.914 & 0.971 & 0.962 & 0.971 & 0.051 \\
\hline
\end{tabular}

4. 3.3 模型结果分析

模型结果表明四个一阶因子均受到一个更高阶潜在因子的影响，其中对高阶潜在因子影 响最高的是比特币技术与安全风险感知, 负荷量为 0.956 , 其次是国家层面的风险感知, 负 荷量为 0.755 , 然后是社会风险, 负荷量为 0.743 , 比特币市场与交易风险排在最后, 为 0.644 。 而这个潜在高阶因子经过分析可以把其命名为比特币投资风险。

另外, 对一阶 CFA 模型和二阶 CFA 模型进行比较, 二阶 CFA 模型中的 RMR 值略超过 0.05, 其它大部分指标的拟合结果相差不大, 基本都能够较好地反映数据量表的结构, 所以由单一 的高阶因子表示基本也可行。

\section{5、结论及政策启示}

本文通过探索性因子分析的方法对比特币风险感知的主要因素进行了研究，确定了国家 层面的风险、比特币技术与安全风险、比特币市场与交易风险、比特币的社会风险这四个潜 在变量，以及政策风险、法律风险、比特币软 硬件技术风险、比特币价格风险、企业跑路风 险、企业服务风险、洗钱风险等 14 个可测变量，并采用结构方程模型对比特币风险感知进行 了实证分析, 通过一阶 $\mathrm{CFA}$ 模型构建得到了各个潜在变量的主要影响因素及影响路径, 也量 化了各个潜在变量之间的相互关系; 在一阶 CFA 基础上探索构建了二阶 CFA 模型, 分析得到 了一个高阶潜在影响因子以及各一阶潜在因子对高阶潜在因子的影响路径。实证研究表明, 比特币风险感知各潜在变量相互影响, 即比特币用户对于比特币的风险感知来自国家层面、 比特币自身层面、市场层面和社会层面，各个层面的风险感知是相互影响的。结合调研数据 和分析结果，本文得到以下政策启示。

第一，加大比特币相关技术的普适教育。研究结果显示比特币技术、安全仍然是用户风 险感知最大的方面, 这表现出用户一方面对于创新技术的关注，另一方面也保持对创新技术 的警戒。为此，借助行业为用户提供比特币相关技术的普适指导和答疑是必要的，通过组织 线上、线下的相关会议或活动，让用户对比特币的硬件技术、软件技术、操作技巧等有进一 步的认识和理解, 引导用户客观、理性学习和投资, 甚至可以根据市场需要和发展考虑适时 开设比特币相关技术 (区块链技术等) 的专业, 从而, 不仅可以避免对技术不了解盲目跟风 引起的心理恐慌; 而且对比特币及其技术的创新发展也起到鼓励作用。

第二，积极推进比特币监管方案的研究，适时制定和出台相关法律法规。从研究结果可 以看出, 用户在国家的法律和政策风险感知方面有较高的警戒性。因为, 一方面, 法律是维 护用户权益和保证比特币市场秩序的重要保障; 另一方面, 比特币作为一种去中心化创新客 体，国家政策的出台从较大程度上影响用户对比特币的关注度和投资策略，所以，用户对于 国家法律和政策的风险感知是必然的。而作为新事物，比特币的监管也会逐步提上日程，鉴 于此，建议相关部门联合行业力量组织若干支专业队伍对比特币相应的技术和应用进行全方 位、多视角的专项深入研究，逐步建立比特币或比特币技术应用相关的法律监管制度，并在 必要的时候通过政府干预手段控制和降低比特币的各种相关风险。

第三，积极寻求国际合作，杜绝利用比特币进行黑色交易。从研究结果看出，社会风险 中人们对于洗黑钱和非法交易的风险感知度明显高于通胀紧缩和替代风险, 说明人们对于比 特币的黑色交易市场存在较高警惕, 担心比特币的生态系统会由于黑色交易而遭到破坏。考 虑到比特币的无国界特点, 国家相关部门可以寻求国际合作, 共同研究和制定反洗钱方案, 抵制利用比特币进行洗黑钱或非法交易的行为。 
第四，加大比特币企业的监管和帮扶力度，规范经营模式。研究结果显示，比特币市场 与交易风险感知方面主要来源于比特币企业和比特币市场价格的跌宕起伏。由于目前国内的 比特币企业都是年轻的创业公司, 公司的人员结构、技术力量、公司管理也都处于起步阶段, 所以，企业的经营、服务也是用户风险感知的主要方面。从这方面讲，企业应该规范内部管 理, 国家相关部门也应当对比特币企业尤其是比特币交易平台进行严格审查和适度帮扶, 一 方面减少企业经营失败风险, 另一方面也可以减少企业到个人的转嫁风险。

\section{致谢}

本文为国家社科基金青年项目《比特币的发展及其风险研究》(编号：14CJY069)的阶段 性成果之一。

\section{References}

[1] M. Möser ,Towards Risk Scoring of Bitcoin Transactions. Financial Cryptography and Data Security LNCS8438, Berlin: Springer-Verlag, pp.16-32,2014.

[2] N. Liu, The puzzle to be solved: the risks, challenges and judicial response to bitcoin ,legality Vision,vol.3,pp. 22-26,2015.

[3] L. Dai, The investment risk analysis of Bitcoin , Communication of Finance and Accounting ,vol.3,pp.3-4,2015.

[4] G. Wang, Z. Y. Feng, Research on the risk characteristics of bitcoin, the latest regulatory trends and policy recommendations, Journal of Finance and Economics,vol.9, pp. 46-49,2013.

[5] F. Brezo, P. Bringas, Issues and Risks Associated with Cryptocurrencies such as Bitcoin. The Second International Conference on Social Eco-Informatics ,Italy: IARIA XPS Press ,pp.20-26, 2012.

[6] T. Moore, N. Christin, Beware the Middleman: Empirical Analysis of Bitcoin-Exchange Risk .Financial Cryptography and Data Security LNCS7859. Berlin:Springer-Verlag,pp.25-33, 2013.

[7] M. L. Wu, Structural equation model: the operation and application of Amos, ChongQing:Chongqing University Press, 2009.

[8] M. N. Li, A brief introduction of structural equation modeling software AMOS and its application in the preparation of test:Graphic \&Basic, TaiBei: Psychology press, 2006. 